\title{
Photoluminescence Properties and Morphologies of Submicron-Sized ZnO Crystals Prepared by Ultrasonic Spray Pyrolysis
}

\author{
Myo Than НтаY *, Minoru Iтон, Yoshio Наsнimoto, and Kentaro Iто \\ Department of Electrical and Electronic Engineering, Faculty of Engineering, Shinshu University, 4-17-1 Wakasato, Nagano 380-8553, \\ Japan
}

\begin{abstract}
Spectral features of the near-band-edge photoluminescence (PL) of submicron-sized $\mathrm{ZnO}$ crystals such as nanoplatelets, nanowires, and nanorods grown by an ultrasonic spray pyrolysis (USP) technique were investigated. The measurements of time-integrated and time-resolved PL spectra were performed in the temperature range of 8-300 K under various excitation densities by using the fourth harmonics $(4.66 \mathrm{eV})$ of a Nd:YAG laser. Exciton-related emission bands were clearly observed in the $\mathrm{ZnO}$ crystals having different morphologies. In nanoplatelets, an emission band originating from radiative recombination of donor-acceptor pairs was also found at around $3.17 \mathrm{eV}$, indicating the existence of acceptor centers. The binding energies of donor and acceptor were about 53 and $200 \mathrm{meV}$, respectively. In nanowires, the intensity of an emission band peaking at $3.32 \mathrm{eV}$ obeyed a quadratic dependency on the density of excitation. This fact shows that an inelastic exciton-exciton scattering process is efficient in the nanowires because of high crystal quality.
\end{abstract}

KEYWORDS: ZnO, nanoplatelets, nanowires, nanorods, ultrasonic spray pyrolysis, time-resolved luminescence measurement, bound exciton complex, two-electron satellite, donor-acceptor pair, inelastic exciton-exciton scattering

\section{Introduction}

$\mathrm{ZnO}$, an environment-friendly material, is a promising candidate for exciton-related optoelectronic devices in the ultraviolet region, and it is also applicable to the devices based on reduced dimensional quantum effect because it can be grown in various crystalline forms with submicron size such as whiskers, nanobelts, nanorods, nanowires, nanoplatelets, and so on. In the previous paper, we reported on direct fabrication of single crystalline $\mathrm{ZnO}$ submicron structures called nanoplatelets, nanowires, and nanorods by an economically viable ultrasonic spray pyrolysis (USP) technique. ${ }^{1)}$ Utilizing this method, significant changes of the crystal morphologies were realized in a controllable manner by adjusting the nature of precursor solution and the growth temperature.

The present paper is concerned with the photoluminescence (PL) properties of submicron-sized $\mathrm{ZnO}$ crystals prepared by USP. In nanoplatelets, the existence of acceptor centers is revealed from the observation of donor-acceptor pair luminescence. An inelastic exciton-exciton scattering process is confirmed in nanowires. Based on these results, we discuss the crystal quality of our submicron-sized $\mathrm{ZnO}$ crystals.

\section{Experimental Procedure}

The $\mathrm{ZnO}$ nanoplatelets, nanowires, and nanorods used in the present study were synthesized by the USP technique. ${ }^{1)}$ The preparation conditions and physical dimensions of the three different types of samples (A, B, and C) investigated in this experiment are summarized in Table I. Surface morphologies of the samples were observed by using a field emission scanning electron microscope (FE-SEM; Hitachi S4100). The chemical composition of each sample was analyzed by an X-ray photoelectron spectroscope (XPS; Surface Science Instruments S-probe 7339, Al K $\alpha 1486.6 \mathrm{eV}$ source).

For time-resolved PL measurements, the fourth harmonics (4.66 eV) of a $Q$-switched Nd:YAG laser was used as an excitation light source. The pulse width, repetition rate, and excitaion energy were $7 \mathrm{~ns}, 10 \mathrm{~Hz}$, and $3 \mathrm{~mJ}$, respectively. An electronic synchronization technique was applied by using a

*E-mail address: s06t215@ shinshu-u.ac.jp digital delay-pulse generator (BNC Model 555). ${ }^{2)}$ The time jitter of the system was $\pm 20 \mathrm{~ns}$. The sample was placed inside a temperature-variable cryostat $(8-300 \mathrm{~K})$. PL from the sample was dispersed by a $0.32 \mathrm{~m}$ monochromator and detected by a gated intensified charge-coupled device (ICCD) camera (Hamamatsu C5909-06). The spectral resolution of the detection system was about $0.5 \mathrm{~nm}$. The excitation power density of laser light was varied using a suitable combination of attenuator meshes. In this paper, the PL spectra measured at a delay time $\left(\tau_{\mathrm{D}}\right)$ of $0 \mathrm{~ns}$ and a gate width $(\Delta t)$ of $1 \mu$ s correspond to the time-integrated spectra, because all the emission bands exhibited decay times that were shorter than $0.1 \mu \mathrm{s}$.

\section{Results and Discussion}

The surface morphology of the three samples is shown in Figs. 1(a) to 1(c), respectively. It is found that the crystallographic directions and the growth orientations of these submicron structures are distributed randomly. Based on the analyses utilizing transmission electron microscopy and electron diffraction, we reported that the crystallographic directions of the wires and rods are in the [0001], [1010], and [11 $\overline{2} 0]$ axes of the wurtzite structure and the crystallographic planes of the nanoplatelets are parallel to the (0001) plane. ${ }^{1)}$ These submicron-sized crystals were confirmed to be monocrystalline.

The results of the chemical composition of samples evaluated by XPS analysis are shown in Table I. It appears that the incorporation of indium impurity in sample $\mathrm{C}$ is fairly higher than that in sample B. This is likely due to enhancement of the diffusion of indium species into the wurtzite crystal lattice at higher growth temperatures. By addition of ammonium acetate in the precursor solutions, doping of nitrogen acceptor impurities in the samples is naturally expectable. However, we could not detect the presence of nitrogen impurities by XPS analysis, since the amount of diffused nitrogen impurities into the samples was much smaller than the detectable level.

The time-integrated PL spectra of sample A (nanoplatelets) taken at $8 \mathrm{~K}$ under various excitation densities, the average power of which were $0.5,1.3,3.2,8.0$, and $20 \mathrm{~mW} / \mathrm{cm}^{2}$, 
respectively, are shown in Fig. 2. At low-density excitation of $0.5 \mathrm{~mW} / \mathrm{cm}^{2}$, a sharp luminescence band located at 3.36 $\mathrm{eV}$ with a full width at half maximum (FWHM) of about 18 $\mathrm{meV}$ is seen, although the free exciton emission, which is located at $3.377 \mathrm{eV},{ }^{3)}$ is not resolved. From the peak energy of $3.36 \mathrm{eV}$, we consider that this band is originated from the recombination of excitons bound to neutral donor $\left(\mathrm{D}^{\circ} \mathrm{X}\right)$ and/or neutral acceptor $\left(\mathrm{A}^{\circ} \mathrm{X}\right)$ impurities, which we call bound exciton complexes (denoted as BECs) as reported in literature. ${ }^{4,5)}$ In sample A, unintentionally diffused hydrogen and nitrogen species could be the chemical origins of these donor and acceptor centers for BECs, respectively. The energy separation between the $\mathrm{D}^{\circ} \mathrm{X}$ and $\mathrm{A}^{\circ} \mathrm{X}$ luminescence is known to be very small $(\leq 10 \mathrm{meV}) .^{5,6)}$ Hence, it is difficult to obtain clearlyresolved $\mathrm{BEC}$ spectra (i.e., identification of the $\mathrm{D}^{\circ} \mathrm{X}$ and $\mathrm{A}^{\circ} \mathrm{X}$ bands) from the samples having randomly orientated crystallographic planes shown in Fig. 1. A very weak emission band, which is considered to be the longitudinal phonon replica of BEC (denoted as BEC-1LO), is also observed at $3.27 \mathrm{eV}$.

Another emission band is seen on the lower energy side of the BEC band. Its peak energy is about $40 \mathrm{meV}$ smaller than that of the BEC band. This is the region where the twoelectron satellite (denoted as TES) transition of the $\mathrm{D}^{\circ} \mathrm{X}$ is expected to take place. ${ }^{3,6,7)}$ In such a transition process, the radiative recombination of a $\mathrm{D}^{\circ} \mathrm{X}$ complex transfers its donor electron from the $1 \mathrm{~s}$ ground state into an excited state such as the $2 \mathrm{~s}, 2 \mathrm{p}$, etc. In the effective-mass approximation, the energy difference between the $\mathrm{D}^{\circ} \mathrm{X}$ complex and its corresponding TES transition is equivalent to the difference between the donor energies in its excited states, which is $3 / 4$ of the donor binding energy. ${ }^{7)}$ Applying this argument, we can obtain the related donor binding energy from the peak position of the TES transition. For sample A, the donor binding energy is estimated to be about $53 \mathrm{meV}$, in good agreement with the earlier experiments. ${ }^{3,6,7)}$

In Fig. 2, a broad PL band appears around $3.17 \mathrm{eV}$ as the density of excitation is increased to $1.3 \mathrm{~mW} / \mathrm{cm}^{2}$. We attribute this band to the luminescence due to the recombination of donor-acceptor pairs (denoted as DAP), since its peak energy shifts towards the higher energy side under higher densities of excitation. This is one of the well-known features of the DAP luminescence. $\left.{ }^{8}\right)$ Teke et al. reported the observation of a clearly resolved DAP band together with its corresponding LO-phonon replicas located successively on the lower energy side by separation of about $72 \mathrm{meV}$ in single crystal $\mathrm{ZnO}{ }^{3}$ ) However, other groups ${ }^{9,10)}$ have observed a broad DAP band that is similar to our case. One reason for such band broadening could be due to the difference in the saturation of close pairs. ${ }^{11)}$ Since the DAP band is composed of a large number of overlaping emission bands originating from the recombination of donor-acceptor pairs with a wide range of intra-pair separation, it is quite difficult to separate these emission bands under steady-state excitation.

The energy of photons resulting from the radiative recombination of DAP is given by ${ }^{12)}$

$$
E\left(r_{\mathrm{DA}}\right)=E_{\mathrm{g}}-E_{\mathrm{D}^{\mathrm{o}}}^{\mathrm{b}}-E_{\mathrm{A}^{\mathrm{o}}}^{\mathrm{b}}+\frac{q^{2}}{4 \pi \varepsilon \varepsilon_{\mathrm{o}} r_{\mathrm{DA}}},
$$

where $E_{\mathrm{g}}$ is the band gap energy, $E_{\mathrm{D}^{\circ}}^{\mathrm{b}}$ and $E_{\mathrm{A}^{\circ}}^{\mathrm{b}}$ are the binding energies of an electron and a hole to their corresponding centers, $q$ is the electric charge of carrier, $\varepsilon$ is the dielectric constant, $\varepsilon_{\mathrm{O}}$ is the permittivity of vacuum, and $r_{\mathrm{DA}}$ is the separation between donor and acceptor centers. The last term of this equation approximates the average inherent Coulomb potential energy of each DAP. It can be alternatively expressed as $\alpha N_{\mathrm{D}}^{1 / 3}$, where the coefficient $\alpha$ is $2.7 \times 10^{-8} \mathrm{eV} \mathrm{cm}$ and the donor concentration $N_{\mathrm{D}}$ of undoped $\mathrm{ZnO}$ single crystal is 1.7 $\times 10^{17} \mathrm{~cm}^{-3}{ }^{7)}$ By using $E\left(r_{\mathrm{DA}}\right) \approx 3.2 \mathrm{eV}, E_{\mathrm{g}} \approx 3.438 \mathrm{eV},{ }^{7)}$ and $E_{\mathrm{D}^{\circ}}^{\mathrm{b}} \approx 53 \mathrm{meV}$ as obtained above, a rough estimation of acceptor binding energy $E_{\mathrm{A}^{\circ}}^{\mathrm{b}}$ is $200 \mathrm{meV}$. This value is close to $195 \mathrm{meV}$ reported by Thonke et al. ${ }^{7)}$

The radiative recombination rate of DAP luminescence is usually expressed by ${ }^{11)}$

$$
W\left(r_{\mathrm{DA}}\right)=W_{\mathrm{o}} \exp \left(-\frac{r_{\mathrm{DA}}}{r_{\mathrm{B}} / 2}\right),
$$

where $W_{\mathrm{o}}$ is the constant and $r_{\mathrm{B}}$ is the Bohr radius of the shallower state. This equation means that the radiative decay time $(=1 / W)$ of low photon energies (large $\left.r_{\mathrm{DA}}\right)$ is longer than that of high photon energies (small $r_{\mathrm{DA}}$ ). The DAP emission spectrum is thus expected to red-shift with increasing the delay time. ${ }^{12)}$ This expectation was also confirmed in our timeresolved experiment on sample A. The result is presented in Fig. 3. No energy shifting in the BEC, TES, and the corresponding LO-phonon bands is found, while the DAP band remarkably red-shifts as the delay time is increased.

From Fig. 3, the decay times of DAP luminescence, at which the intensity is decreased to $50 \%$ of the initial value, are roughly estimated to be $10 \mathrm{~ns}$ and $100 \mathrm{~ns}$ near $3.2 \mathrm{eV}$ and $2.9 \mathrm{eV}$, respectively. The value of $10 \mathrm{~ns}$ is somewhat longer than the value $(5.5 \mathrm{~ns})$ of DAP luminescence at $3.232 \mathrm{eV} \mathrm{ob-}$ served by Xiong et al. ${ }^{13)}$ The decay times of BEC and TES bands are less than $10 \mathrm{~ns}$, which are much longer than those reported in references 3 and 14. This could be due to the difference in the sample condition and/or excitation intensity.

The observation of DAP band is a clear proof of the existence of acceptor centers together with donor centers in this sample. If considerable amount of unintentionally incorporated donor impurities such as interstitial zinc or hydrogen species in the sample can be suppressed during the crystal growth, the formation of $p$-type conductivity is expectable by using our low-cost USP technique. In fact, Vlasenflin and Tanaka have very recently reported on the formation of $p$-type conduction in $\mathrm{ZnO}: \mathrm{N}, \mathrm{P}$ films prepared by the USP technique, after the post-annealing treatment. ${ }^{15)}$

Figure 4 shows the temperature dependent PL spectra of sample A measured under the excitation density of 8 $\mathrm{mW} / \mathrm{cm}^{2}$. Due to thermal dissociation of excitons from the complexes, the intensity of BEC band decreases rapidly as the lattice temperature increases. When the lattice temperature becomes higher than $100 \mathrm{~K}$, the BEC band overlaps with the TES band. The disappearance of the TES band is fairly similar to that of the BEC band. This corresponds to the fact that the TES band cannot exist without its primitive BEC emission.

The time-integrated PL spectra of sample B (nanowires) taken under various excitation densities at $8 \mathrm{~K}$ are shown in Fig. 5. A sharp $3.36 \mathrm{eV}$ band arising from the BEC is dominant at low-density excitation of $0.5 \mathrm{~mW} / \mathrm{cm}^{2}$. In this sample, indium impurities could be the chemical origin for the neutral donor centers. An emission band (denoted as P) centered at $3.32 \mathrm{eV}$ emerges as the excitation density is increased. A very 
weak and broad band corresponding to the LO-phonon replica is also observable at around $3.27 \mathrm{eV}$. The DAP band observed in sample A (nanoplatelets) does not appear in this sample (nanowires). This is probably because the incorporation of indium impurities as donor centers suppresses the formation of acceptor centers, leading to the disappearance of the DAP emission. By assuming that the spectral shape of BEC and $\mathrm{P}$ bands has a Gaussian distribution, the dependence of individual PL intensities on the excitation density was determined. The result is presented in Fig. 6. The BEC band follows a linear dependence on the excitation intensity, while the $\mathrm{P}$ band exhibits a superlinear relationship with the exponent of 2 . This kind of quadratic dependence of the $\mathrm{P}$ band is well explained in terms of an inelastic scattering process between two excitons, in which one exciton recombines radiatively while the other is scattered under energy and momentum conservation into a higher state $(n>1)$ or into the continuum. Another distinct feature of the inelastic exciton-exciton scattering process is that the emission maximum of the luminescence band shifts towards the lower energy side with the increase of excitation density. ${ }^{16)}$ This behavior was also confirmed in sample B, as depicted by a curve with filled triangles in Fig. 6. The observation of the $\mathrm{P}$ band has been reported in high-quality epitaxial thin films. ${ }^{17,18)}$ It can, therefore, be said that the crystal quality of nanowires grown by USP technique is high enough or, at least, comparable to those prepared by the epitaxial technique.

Figure 7 shows the excitation-density dependent PL spectra of sample $\mathrm{C}$ (nanorods) at $8 \mathrm{~K}$. At low-density excitation of $0.5 \mathrm{~mW} / \mathrm{cm}^{2}$, an emission band is observed at around 3.36 $\mathrm{eV}$. By comparing this band with the BEC band in sample $\mathrm{B}$ [spectrum (a) in Fig. 5], it is recognized that the FWHM broadens to about 3 times $(60 \mathrm{meV})$, with a band tailing on the low-energy side. As the excitation density increases, the emission maximum of the band shifts towards the lower energy side, as in the case of the $\mathrm{P}$ band in sample B. However, the quadratic dependency on the excitation density was not obvious. The reduction of such nonlinear optical process in sample $\mathrm{C}$ is related by two facts: the incorporation of indium impurities is too much (about 1 at. \%) and the growth rate is relatively fast. We found that sample $\mathrm{C}$ is grown in bulky structure compared to sample B [see Figs. 1 (b) and 1 (c)], although the growth period is the same. These facts suggest that the crystal lattice of bulky nanorods in sample $\mathrm{C}$ is largely disordered due to a large amount of indium impurities and deformed due to the formation of dislocations and defects during the rapid crystal growth. Hence, there is a strong possibility that the excitons are quickly captured by lattice imperfections in sample $\mathrm{C}$, resulting in the reduction of the exciton-exciton scattering process.

\section{Conclusions}

The spectral features of the near-band-edge PL of submicron-sized $\mathrm{ZnO}$ crystals grown by USP were studied by using a time-resolved luminescence technique. In our growth technique, the morphologies of crystals were influenced by the incorporation of indium catalyst and the growth temper- ature. The difference in PL spectra of submicron-sized $\mathrm{ZnO}$ crystals were mainly due to the indium impurity content. In nanoplatelets, a sharp emission band due to BECs, centered at $3.36 \mathrm{eV}$, was observed together with its TES emission band. The nanoplatelets also exhibited a broad band at around 3.17 $\mathrm{eV}$, which was identified as the emission originating from the radiative recombination of DAPs. The presence of DAP emission indicates the existence of acceptor centers together with donor centers in the nanoplatelets. The binding energies of donor and acceptor were estimated to be about 53 and 200 $\mathrm{meV}$, respectively. The nanowires exhibited the $\mathrm{P}$ band originating from an inelastic exciton-exciton scattering process. Its emission intensity enhanced quadratically against the excitation density. The band broadening was found in PL spectra of nanorods. From the observation of exciton-related luminescence bands described above, it is concluded that the crystal quality of submicron-sized $\mathrm{ZnO}$ crystals (especially, nanoplatelets and nanowires) prepared by USP technique is good enough for applications such as optical devices using reduced dimensional quantum effect of excitons.

\section{Acknowledgments}

One of the authors (M. T. Htay) would like to express his gratitude to the Ministry of Education, Culture, Sports, Science, and Technology of Japan for a grant of postgraduate scholarship. Special thanks are also due to Mr. Daisuke Iri and Mr. Tsuyoshi Katagiri for their great help in the PL measurements.

1) M. T. Htay, Y. Hashimoto, and K. Ito: Jpn. J. Appl. Phys. 46 (2007) 440.

2) M. Itoh and T. Sakurai: Phys. Rev. B 73 (2006) 235106.

3) A. Teke, Ü. Özgür, S. Doğan, X. Gu, H. Morkoç, B. Nemeth, J. Nause, and H. O. Everitt: Phys. Rev. B 70 (2004) 195207.

4) D. C. Reynolds, C. W. Litton, and T. C. Collins: Phys. Rev. 140 (1965) A1726.

5) J. Gutowski, N. Presser, and I. Broser: Phys. Rev. B 38 (1988) 9746.

6) B. K. Meyer, H. Alves, D. M. Hofmann, W. Kriegseis, D. Forster, F. Bertram, J. Christen, A. Hoffmann, M. Straßburg, M. Dworzak, U. Haboeck, and A. V. Rodina: Phys. Status Solidi B 241 (2004) 231.

7) K. Thonke, Th. Gruber, N. Teofilov, R. Schönfelder, A. Waag, and R. Sauer: Physica B 308-310 (2001) 945.

8) C. F. Klingshirn: Semiconductor Optics (Springer-Verlag, Berlin, Heidelberg, 1995) Chap. 15

9) X. H. Wang, B. Yao, D. Z. Shen, Z. Z. Zhang, B. H. Li, Z. P. Wei, Y. M. Lu, D. X. Zhao, J. Y. Zhang, X. W. Fan, L. X. Guan, and C. X. Cong: Solid State Commun. 141 (2007) 600.

10) D. H. Chi, L. T. T. Binh, N. T. Binh, L. D. Khanh, and N. N. Long: Appl. Surf. Sci. 252 (2006) 2770.

11) D. G. Thomas, J. J. Hopfield, and W. M. Augustyniak: Phys. Rev. 140 (1965) A202.

12) J. I. Pankove: Optical Processes in Semiconductors (Dover, New York, 1971) Chap. 6.

13) G. Xiong, K. B. Ucer, R. T. Williams, J. Lee, D. Bhattacharyya, J. Metson, and P. Evans: J. Appl. Phys. 97 (2005) 043528.

14) T. Koida, S. F. Chichibu, A. Uedono, A. Tsukazaki, M. Kawasaki, T. Sota, Y. Segawa, and H. Koinuma: Appl. Phys. Lett. 82 (2003) 532.

15) T. H. Vlasenflin and M. Tanaka: Solid State Commun. 142 (2007) 292.

16) C. Klingshirn: Adv. Mater. Opt. Electron. 3 (1994) 103.

17) P. Zu, Z. K. Tang, G. K. L. Wong, M. Kawasaki, A. Ohtomo, H. Koinuma, and Y. Segawa: Solid State Commun. 103 (1997) 459.

18) H. J. Ko, Y. F. Chen, Z. Zhu, T. Yao, I. Kobayashi, and H. Uchiki: Appl. Phys. Lett. 76 (2000) 1905. 

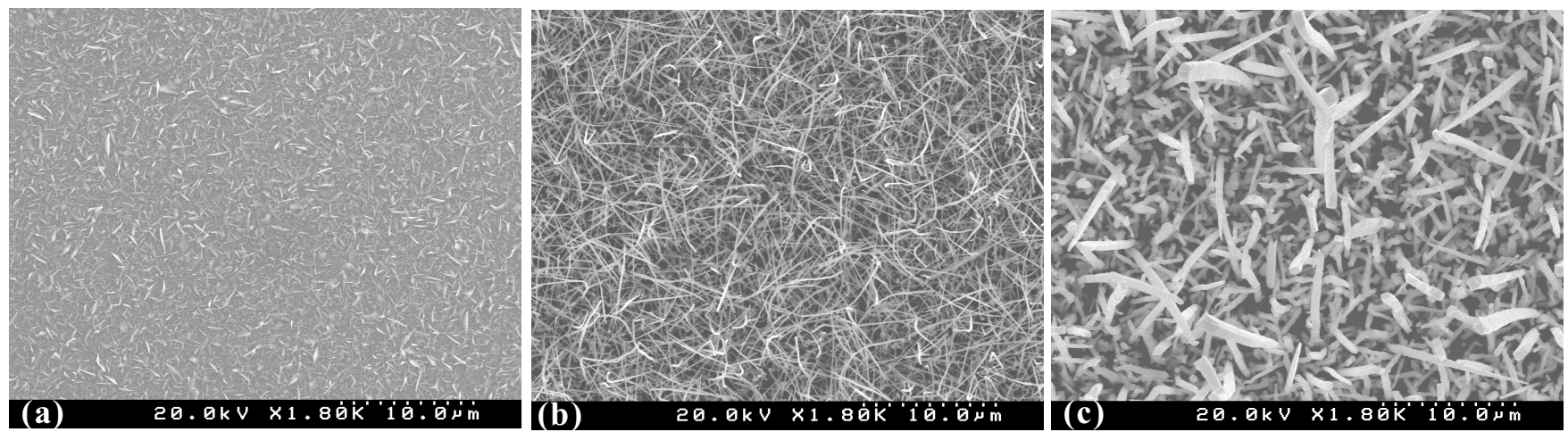

Fig. 1. FE-SEM images showing the surface morphologies of (a) nanoplatelets, (b) nanowires, and (c) nanorods, respectively.

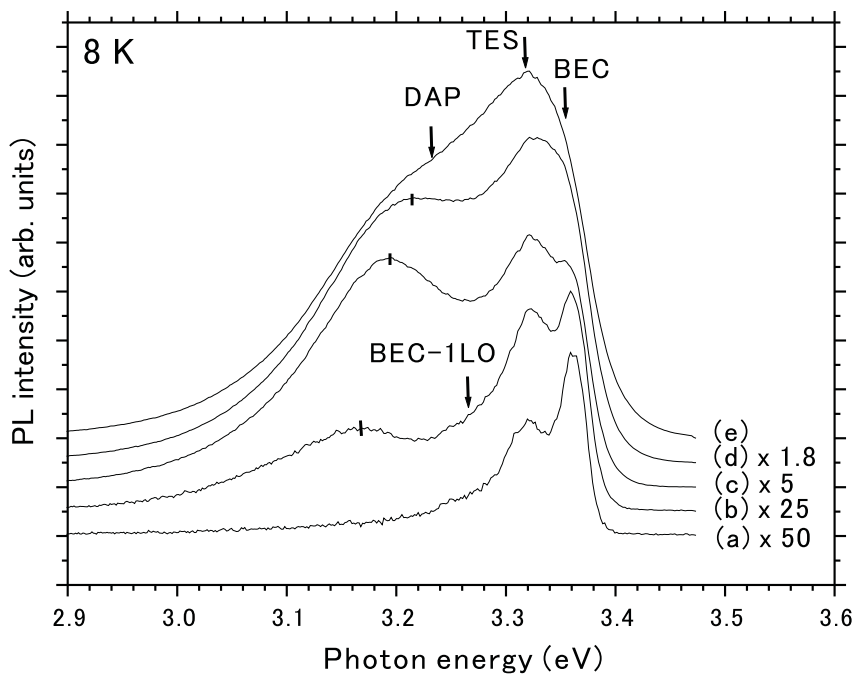

Fig. 2. Time-integrated PL spectra of sample A measured at $8 \mathrm{~K}, \tau_{\mathrm{D}}=0$ $\mathrm{ns}$, and $\Delta t=1 \mu \mathrm{s}$ under different excitation densities of (a) 0.5 , (b) 1.3 , (c) 3.2 , (d) 8.0 , and (e) $20 \mathrm{~mW} / \mathrm{cm}^{2}$, respectively. The intensities of the spectra obtained at low-excitation denisty are multiplied by an arbitrary factor indicated in the figure for visual enhancement.

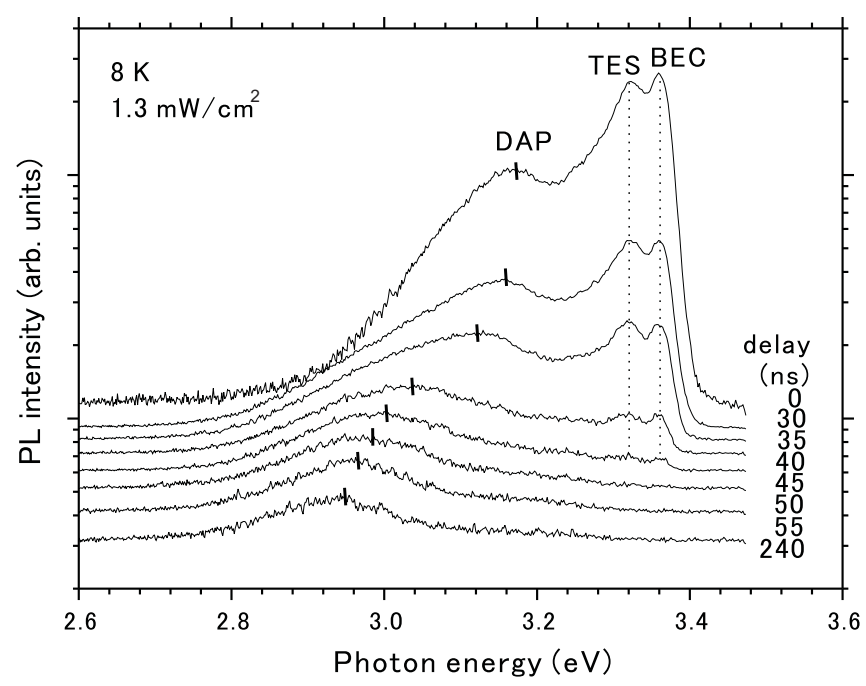

Fig. 3. Time-resolved PL spectra of sample A measured at different delay times and a constant gate width of $40 \mathrm{~ns}$ under the excitation density of 1.3 $\mathrm{mW} / \mathrm{cm}^{2}$ at $8 \mathrm{~K}$. The emission intensity is presented in a logarithmic scale. 


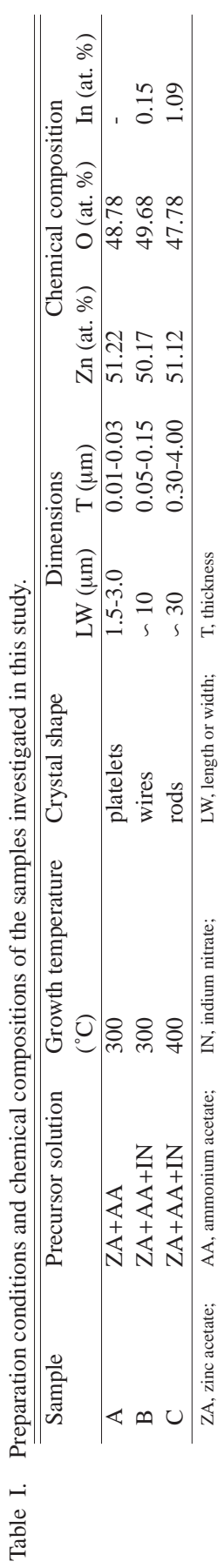




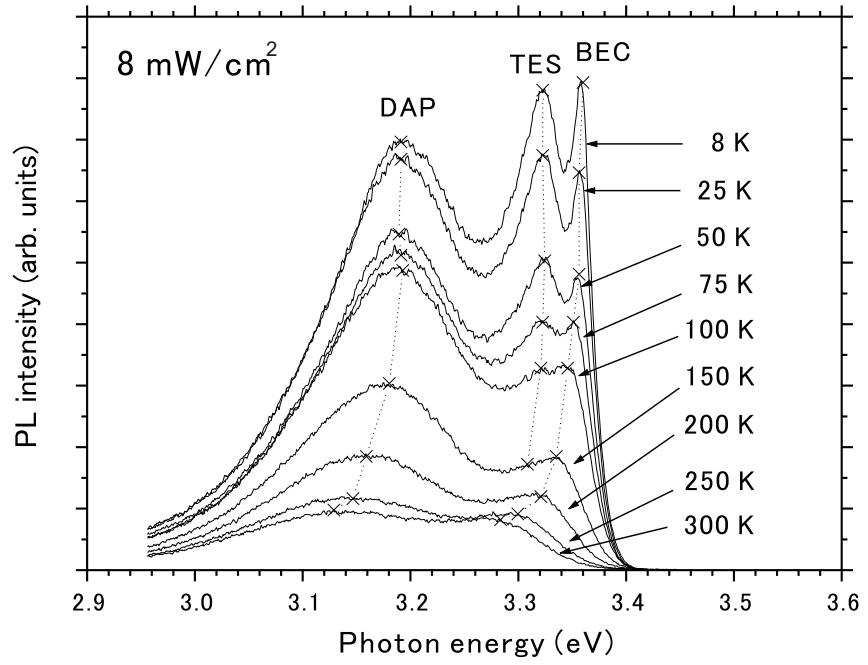

Fig. 4. Temperature dependent PL spectra of sample A measured at $\tau_{\mathrm{D}}=$ $0 \mathrm{~ns}$ and $\Delta t=1 \mu \mathrm{s}$ under the excitation density of $8 \mathrm{~mW} / \mathrm{cm}^{2}$.

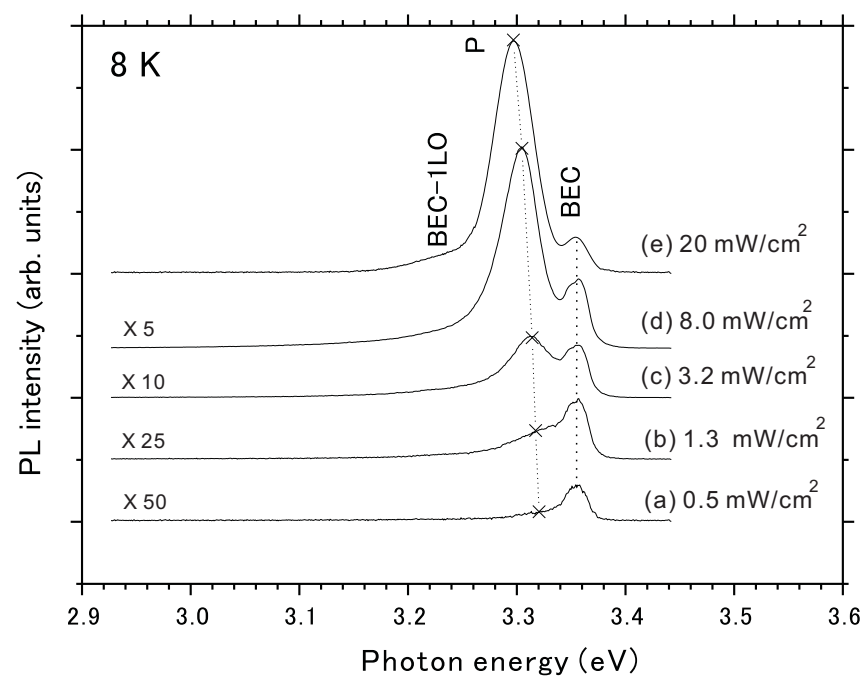

Fig. 5. Excitation-density dependent PL spectra of sample B taken at $8 \mathrm{~K}$ under $\tau_{\mathrm{D}}=0 \mathrm{~ns}$ and $\Delta t=1 \mu \mathrm{s}$. The intensities of the spectra obtained at low-excitation density are multiplied by an arbitrary factor indicated in the figure for visual enhancement. 


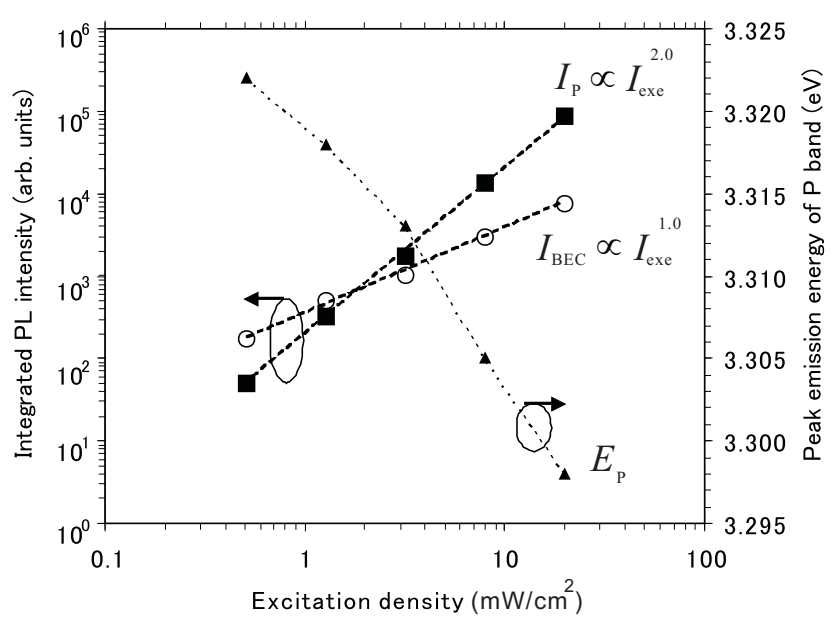

Fig. 6. A log-log plot of the integrated PL intensity for each band against

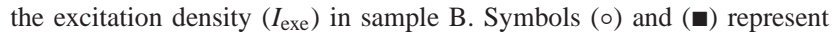
the integrated PL intensity of the BEC band $\left(I_{\mathrm{BEC}}\right)$ and P band $\left(I_{\mathrm{P}}\right)$, respectively. Symbol $(\boldsymbol{\Lambda})$ is the emission peak $\left(E_{\mathrm{p}}\right)$ of the $\mathrm{P}$ band

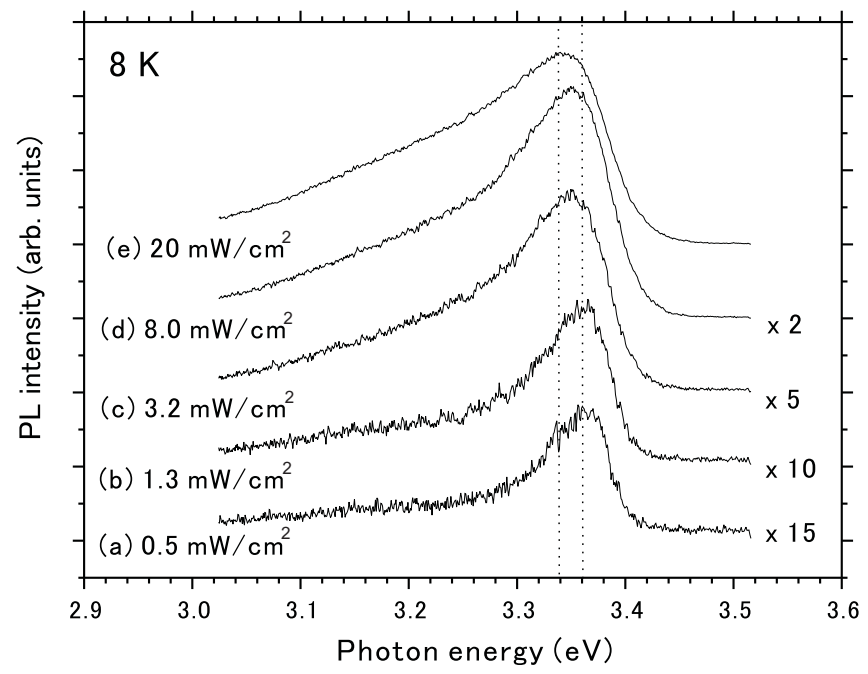

Fig. 7. Time-integrated PL spectra of sample $\mathrm{C}$ measured at $8 \mathrm{~K}, \tau_{\mathrm{D}}=0$ $\mathrm{ns}$, and $\Delta t=1 \mu$ s under different excitation densities. The intensities of the spectra obtained at low-excitation density are multiplied by an arbitrary factor indicated in the figure for visual enhancement. 